\title{
Controle do Intervalo QT para Prevenção de Torsades de Pointes Durante uso de Hidroxicloroquina e/ou Azitromicina em Pacientes com COVID 19
}

\section{QT Interval Control to Prevent Torsades de Pointes during Use of Hydroxychloroquine and/or Azithromycin in Patients with COVID-19}

\author{
Tan Chen Wu, ${ }^{1}$ Luciana Sacilotto, ${ }^{\circledR}$ Francisco Carlos da Costa Darrieux, ${ }^{1}$ Cristiano Faria Pisani, ${ }^{\circledR}$ Sissy Lara de \\ Melo, ${ }^{1 \oplus}$ Denise Tessariol Hachul, ${ }^{1}$ Mauricio Scanavacca ${ }^{1 \odot}$ \\ Universidade de São Paulo Faculdade de Medicina Hospital das Clínicas Instituto do Coração - Arritmia, ${ }^{1}$ São Paulo, SP - Brasil
}

\section{Introdução}

Em dezembro de 2019, foram relatados os primeiros casos da doença causada pelo novo coronavírus (COVID-19), originários de Wuhan, China. ${ }^{1}$ Desde a declaração de pandemia em março de 2020 por Organização Mundial da Saúde (OMS), com disseminação intercontinental, vivenciamos intensa busca por um tratamento seguro e eficaz.

Estudos in vitro demonstraram algum efeito da cloroquina contra o novo coronavírus, ${ }^{3}$ intermediada pela glicosilação dos receptores celulares de SARS-CoV e pelo aumento do $\mathrm{pH}$ endossômico, bloqueando a invasão celular pelo vírus. ${ }^{4}$ Além dessa atividade antiviral, a cloroquina, tradicionalmente um imunomodulador, emergiu como promissora no tratamento da pneumonia que se instala em torno de uma semana após o início dos sintomas. ${ }^{5}$

A hidroxicloroquina (HCQ), derivada da cloroquina, tem efeitos terapêuticos semelhantes e menos efeitos adversos, sendo amplamente utilizada em doenças autoimunes. Os primeiros ensaios clínicos com a HCQ, no tratamento do COVID-19, reforçaram um aparente benefício e encorajaram a sua aprovação para estudos clínicos por órgãos regulatórios nacionais e internacionais. ${ }^{6-8}$

O macrolídeo azitromicina (AZ), ainda por mecanismo incerto, demonstrou ser efetivo quando iniciado precocemente em pacientes com infecções respiratórias graves. ${ }^{9}$ Embora estas medicações tenham um adequado perfil de segurança em diversas situações clínicas, ambos bloqueiam o canal

\section{Palavras-chave}

Coronavirus/complicações, COVID-19, Pandemia, Torsades Pointes, Taquicardia Ventricular, Hidroxicloroquina/ uso terapêutico, Azitromicina/uso terapêutico; Arritmias.

\author{
Correspondência: Tan Chen Wu • \\ Universidade de São Paulo Faculdade de Medicina Hospital das Clínicas \\ Instituto do Coração - Arritmia - Av. Dr. Eneas de Carvalho Aguiar, 44. CEP \\ 05403-000, São Paulo, SP - Brasil \\ E-mail: tan_chen@bol.com.br, tanchen.cardio@gmail.com \\ Artigo recébido em 27/04/2020, revisado em 29/04/2020, aceito em \\ 29/04/2020
}

DOI: https://doi.org/10.36660/abc.20200389 de potássio hERG, podendo prolongar a repolarização ventricular e causar torsades de pointes $(\mathrm{TdP}) ;^{10-11}$

O subgrupo da população com maior risco de eventos potencialmente fatais são os pacientes com múltiplas comorbidades ou em cuidados intensivos, que estarão expostos a interações medicamentosas e/ou a distúrbios eletrolíticos, além dos portadores da síndrome do QT longo congênito, que podem necessitar do tratamento (1:2000 indivíduos). ${ }^{12}$ A avaliação do risco antes e o monitoramento do intervalo QTc durante são fundamentais para prevenção de eventos arrítmicos.

Giudicessi et al., ${ }^{13}$ divulgaram uma diretriz institucional da Mayo Clinic, para a segurança dos pacientes em uso de HCQ e/ou AZ. ${ }^{13}$ O American College of Cardiology apresentou uma sugestão para controle do intervalo QT e prevenção de arritmias ventriculares em pacientes que participam do protocolo HQC/AZ para tratamento do COVID-19. ${ }^{14}$ 0 Núcleo de Arritmias do Instituto do Coração formulou um protocolo institucional a fim de contribuir para o uso consciente dessas medicações durante o surto de infeção por COVID-19.

\section{Definição}

O intervalo QT é a medida da duração do início do complexo QRS até o final da onda T e é modulado pela frequência cardíaca (Figura 1). Quando prolongado, está associado a maior risco de ocorrência de arritmias ventriculares polimórficas e TdP (Figura 2). ${ }^{15}$ A medida do intervalo QT deve ser corrigida pela frequência cardíaca (QTC) e, na população adulta, é considerada normal quando $\leq 440$ ms em homens e $\leq 460$ ms em mulheres. ${ }^{16}$

\section{Como Medir o Intervalo QTC}

O intervalo QT pode ser medido pelo método de tangente (Figura 3) ou visual (quando o final da onda $T$ for de fácil definição), preferencialmente em derivações DII ou V5. ${ }^{17}$

A correção pela frequência cardíaca pode ser feita pela fórmula de Bazett, considerando-se o RR precedente ao intervalo QT medido (QTc = intervalo QT/raiz quadrada do intervalo RR). Essa fórmula está disponível em calculadoras de sites (QTc calculadora) ou em Apps (por exemplo: EP Mobile ou MedCalX). 


\section{Ponto de Vista}

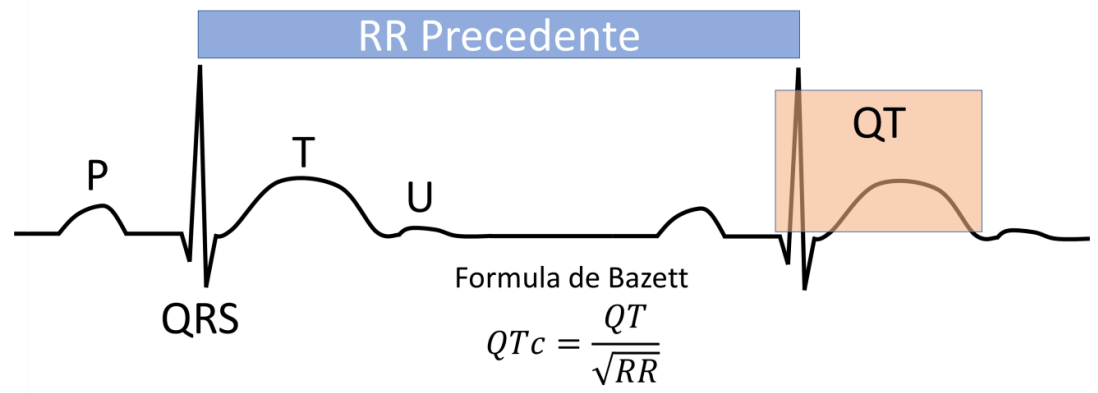

Figura 1 - Intervalo QT. Fonte: Acervo InCor HCFMUSP
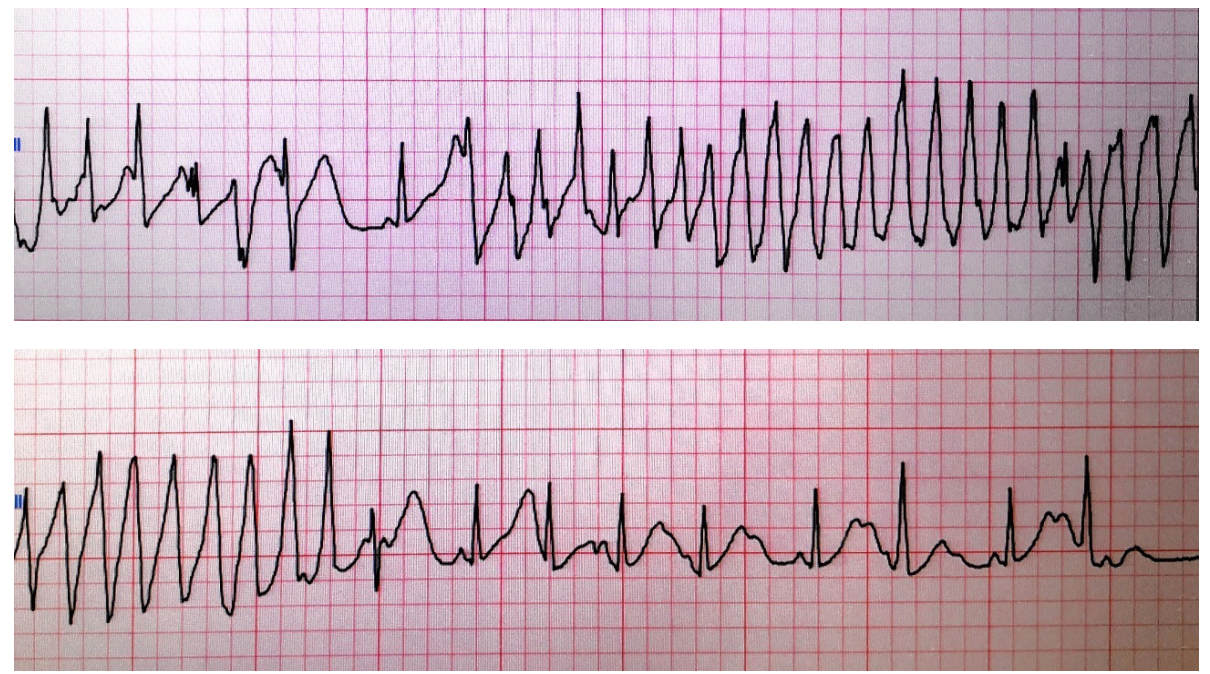

Figura 2 - QT longo com torsades de pointes, Fonte: Acervo InCor HCFMUSP

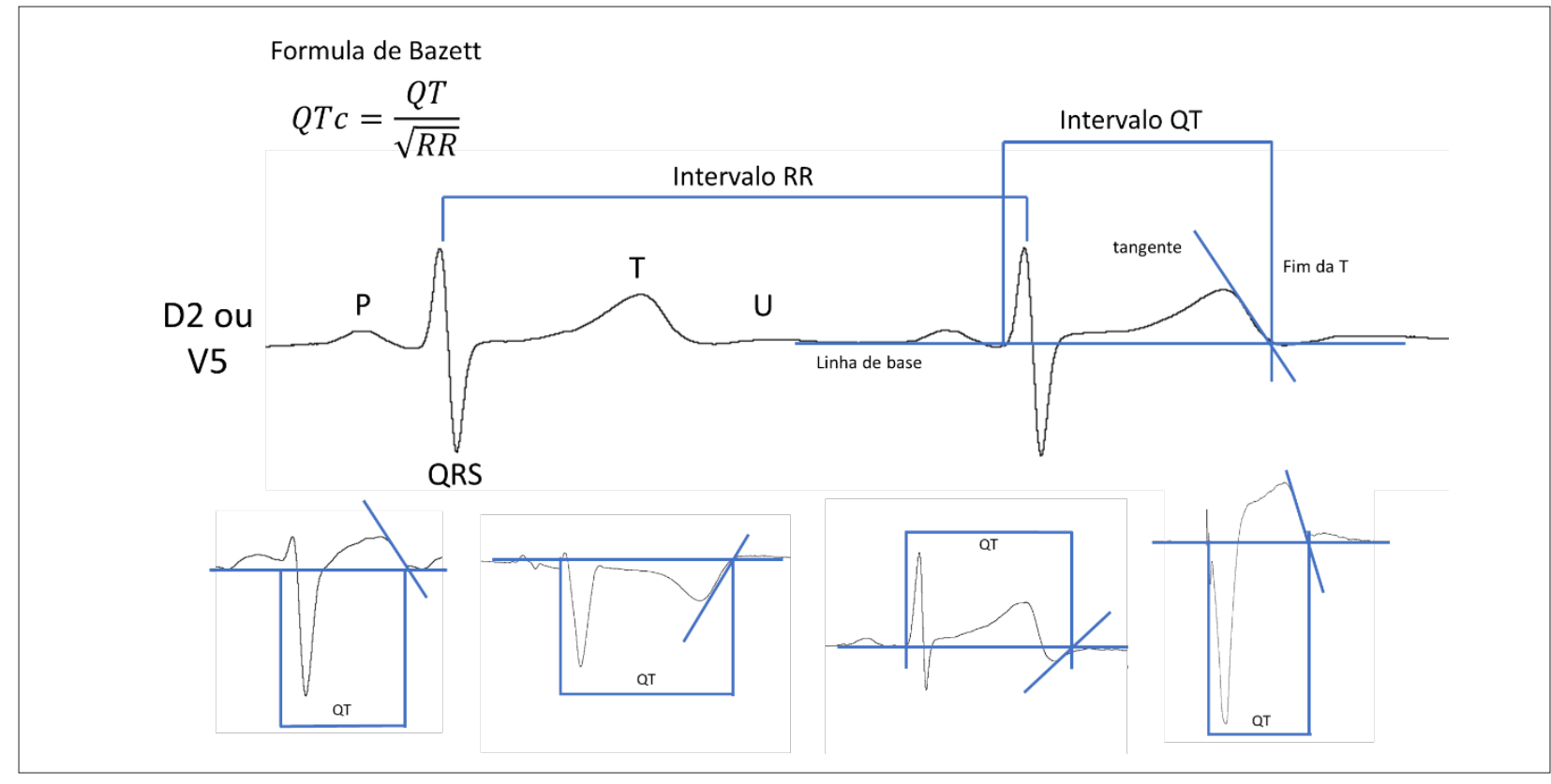

Figura 3 - Exemplos de determinação do intervalo QT pelo método de tangente. Fonte: Acervo InCor HCFMUSP, 


\section{Monitoramento do intervalo QTc durante o tratamento com HCQ/AZ}

Após avaliação do ECG inicial, os pacientes podem ser estratificados conforme o risco de desenvolver TdP, em menor risco (grupo verde), risco intermediário (grupo azul), risco intermediário a elevado (grupo laranja) e alto risco (grupo vermelho).

A monitorização após início do tratamento pode ser feita pelo ECG convencional de 12 derivações, ECG apenas com derivações periféricas, pela telemetria ou por outros dispositivos remotos, para nessa situação peculiar de pandemia, minimizar a exposição de profissionais de saúde e de equipamentos ao vírus. Sugerimos que a frequência de monitorização eletrocardiográfica e o método (ECG, telemetria ou dispositivos) sejam determinados pelo risco do paciente, baseado em um QTc inicial (admissional). A figura 4 esquematiza o modelo de controle proposto.

Quanto à avaliação de risco inicial do paciente para o tratamento pela medida do QT basal em ECG de 12 derivações:

\begin{tabular}{lc}
\hline QTc $450 \mathrm{~ms}$ & Liberado para uso \\
$450 \mathrm{~ms}<$ QTc $\leq 470 \mathrm{~ms}$ & Cautela ou apenas uso em regime hospitalar \\
$470 \mathrm{~ms}<$ QTc $<500 \mathrm{~ms}$ & Evitar ou somente uso hospitalar com telemetria \\
QTc $500 \mathrm{~ms}$ & Evitar, considerando o risco/beneficio \\
\hline
\end{tabular}

Em casos de dúvidas ou medições limítrofes para maior risco ao longo do tratamento, pode-se optar pelo uso isolado da HCQ ou da AZ ou também pelo uso escalonado da HCQ, seguido da AZ, sob monitorização. Sugere-se decisão compartilhada com a equipe de cardiologia ou arritmia do hospital.
Quando repetir o ECG durante o tratamento hospitalar de acordo com o QTc prévio

\begin{tabular}{|c|c|}
\hline QTc $450 \mathrm{~ms}$ & No $2^{\circ} \mathrm{dia}$ \\
\hline $450 \mathrm{~ms}<\mathrm{QTc} \leq 470 \mathrm{~ms}$ & No $2^{\circ} \mathrm{dia}$ \\
\hline $470 \mathrm{~ms}<\mathrm{QTc}<500 \mathrm{~ms}$ & No $2^{\circ}$ dia e no $4^{\circ}$ dia \\
\hline QTC $500 \mathrm{~ms}$ & $\begin{array}{c}\text { Em } 4 \text { a } 8 \text { horas após a primeira dose } \\
\text { e diariamente }\end{array}$ \\
\hline
\end{tabular}

Intensificar o controle nas seguintes condições:

- Se houver fatores de risco associados (Tabela 1).

- Na presença de complicações cardiovasculares como miocardite e isquemia miocárdica.

Obs.: Sugestão de modelo para Lista de checagem prétratameto e controle apresentadas nas Figuras 5 e 6.

\section{Sinais de alerta}

- Aumento do QTC > 60 ms e/ou mais de 10\% em relação ao ECG basal.

- QTC acima de 520 ms - avaliar suspensão do tratamento depois de serem suspensos outros fármacos (dispensáveis e com efeito sinérgico sobre o QTC) ou distúrbio eletrolítico.

- Necessidade de adicionar medicações que prolongam o intervalo QT, conforme evolução clínica do paciente.

- Presença de arritmia ventricular e/ou bradicardia associada -> escolher os fármacos que podem ser suspensos conforme o balanço risco vs. benefício. Nessas situações, há necessidade de manter o paciente sob telemetria contínua.

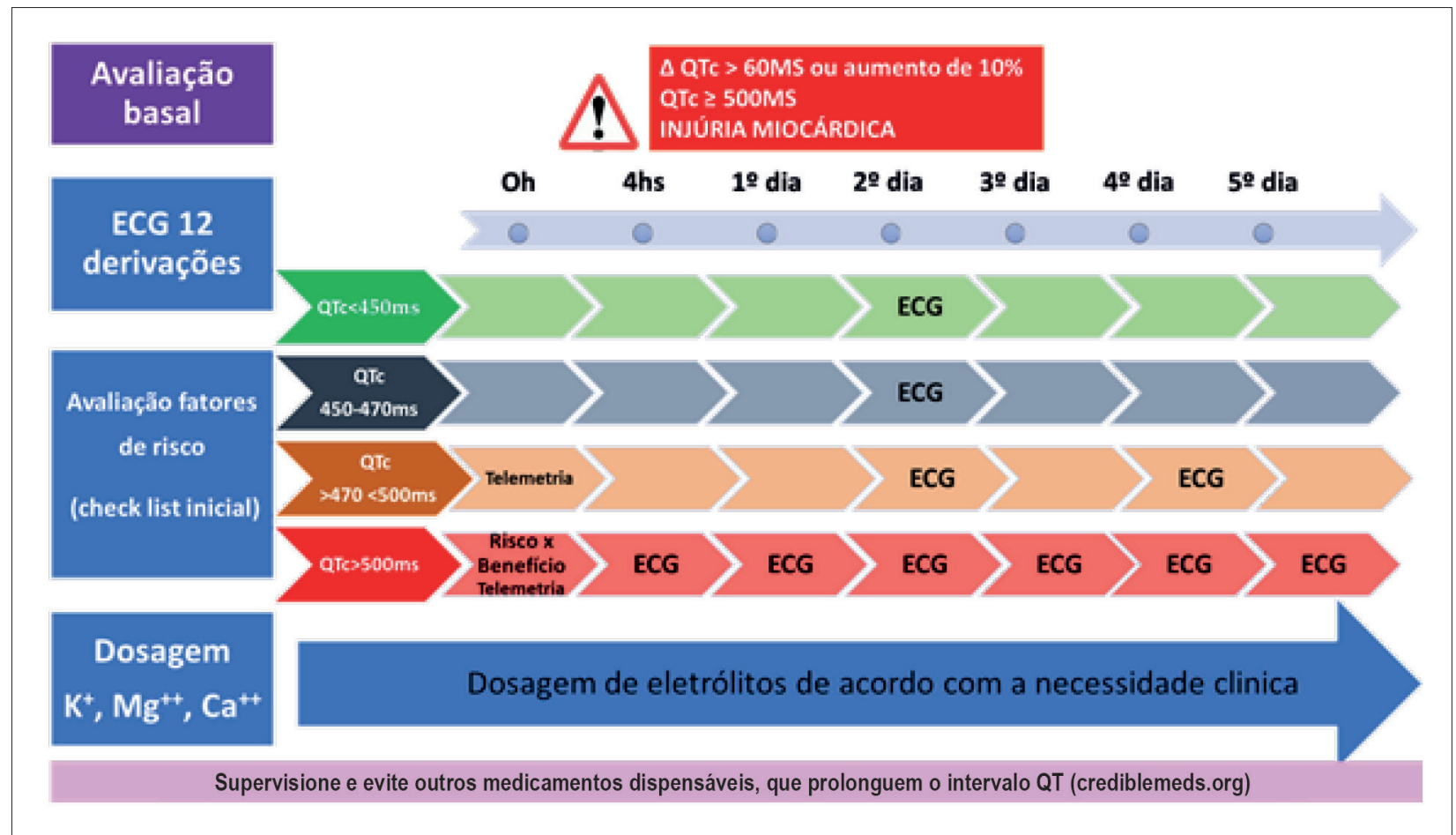

Figura 4 - Esquema sugerido de controle do tratamento $\mathrm{HCQ}$ e/ouAZ 


\section{Ponto de Vista}

\section{Tabela 1 - Fatores de risco para prolongamento de QT e TdP. (18)}

- Idade > 65 anos

- Mulheres

- Distúrbios eletrolíticos (hipocalcemia, hipocalemia, hipomagnesemia)

- Uso concomitante de mais medicações que prolongam QT (crediblemeds.org)

- Insuficiência coronariana aguda

- Insuficiencia cardiaca crônica ou FEVE < 40\%

- Bradicardia, bloqueio de ramo

- Cardiomiopatia hipertrófica

- Síndrome do QT longo congênito ou outra susceptibilidade genética

- Diabetes mellitus

- Insuficiência renal crônica dialítica

- Anorexia ou inanição

- Hipoglicemia

- Feocromocitoma

- Pós-parada cardiorrespiratória recente

- Pós-hemorragia subaracnóidea, acidente vascular cerebral ou traumatismo crânio encefálico ( ${ }^{\circ}$ semana).

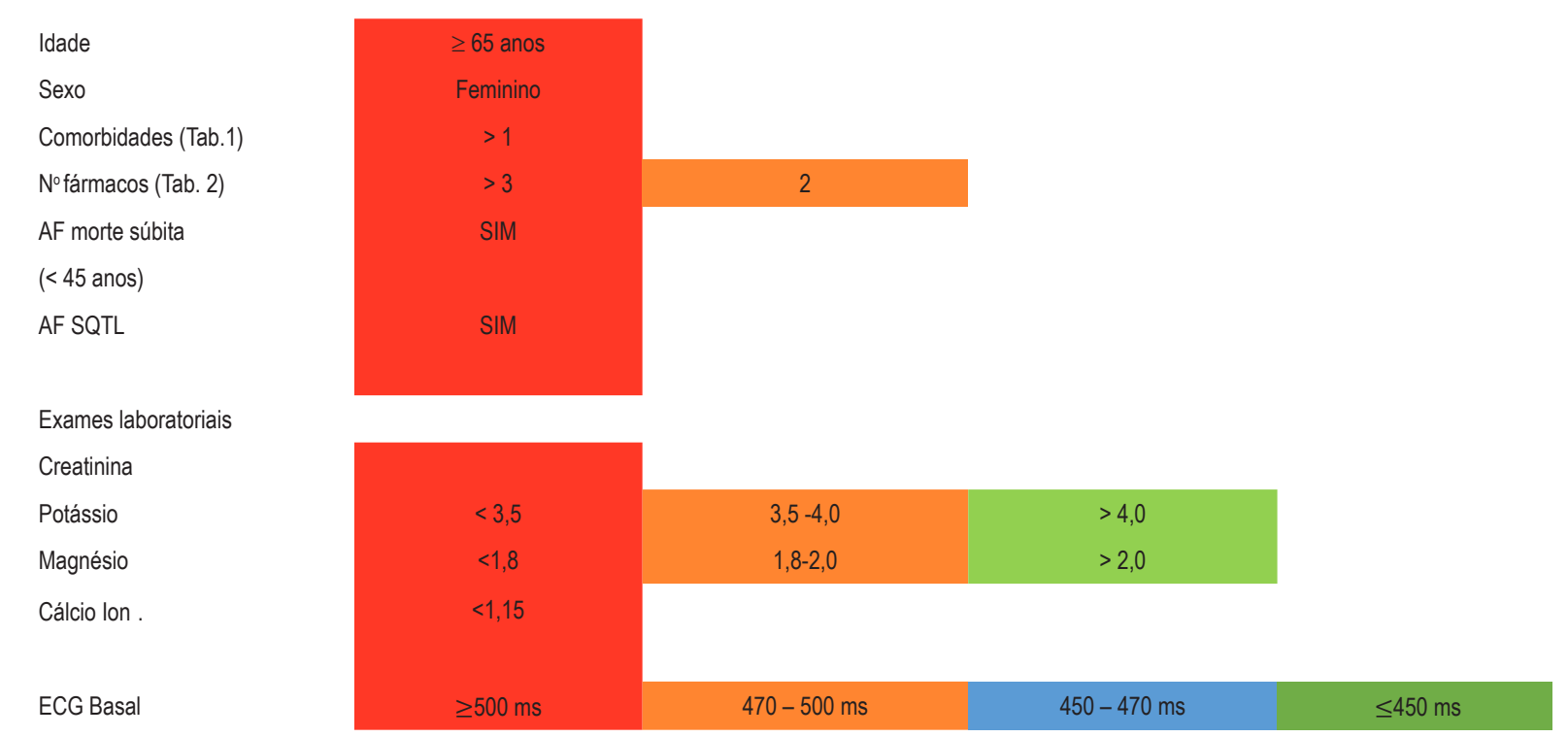

Figura 5 - Lista de checagem PRÉ-tratamento. $A F=$ antecedente familiar; $S Q T L$ = síndrome do $Q T$ longo congênito

Em vermelho: atenção às condições de risco; em laranja: moderado risco; em verde: baixo risco ou alvo desejável

Comorbidades $($ Tab.1)*

№ fármacos (Tab. 2)

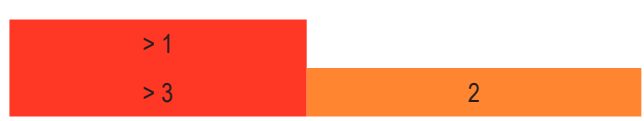

Exames laboratoriais

Creatinina

Potássio

Magnésio

Cálcio lon .

ECG Controle

Variação do QT

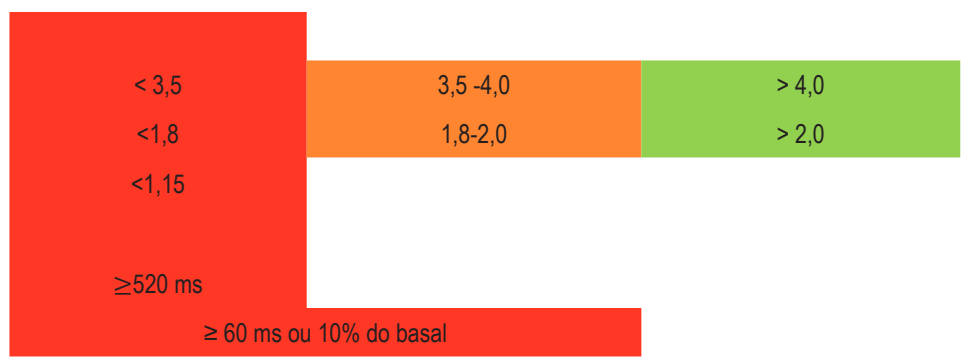

Figura 6 - Lista de checagem CONTROLE:

* Condições clínicas e metabólicas durante a evolução clínica: injurias miocárdicas entre outras. 


\section{Cuidados adicionais para prevenção de TdP}

\section{Quanto ao controle de eletrólitos na admissão:}

A dosagem de cálcio, potássio e magnésio, fundamentais na estabilidade da repolarização ventricular, deve ser realizada para todos os pacientes elegíveis para o tratamento com HCQ/AZ.

$$
\begin{aligned}
& \text { - Manter } \mathrm{K}^{+}>4,0 \\
& \text { - Manter } \mathrm{Mg}^{++}>2,0 \\
& \text { - Evitar hipocalcemia }
\end{aligned}
$$

Obs.: mesmo em pacientes com nível sanguíneo normal, recomenda-se manter suplementação empírica de magnésio via oral, exceto naqueles com insuficiência renal ( $\mathrm{ClCr}<30 \mathrm{ml} / \mathrm{min})$.

\section{Quanto ao controle de eletrólitos na evolução:}

A rotina de monitorização de eletrólitos deve ser realizada a critério clínico, sempre que houver necessidade de ajustes para manter os níveis ideais ou desejáveis durante o tratamento, principalmente nos pacientes com intervalo QTc inicial > $470 \mathrm{~ms}$.

\section{Quanto ao uso de medicações concomitantes:}

Deve-se evitar a prescrição de outros medicamentos, não essenciais, que prolonguem o intervalo QT. Inúmeras drogas, utilizadas habitualmente em pacientes internados, podem bloquear o canal hERG, prolongar o tempo de repolarização ventricular e facilitar a ocorrência de TdP. ${ }^{18}$ A supervisão de farmacêuticos é desejável sempre que possível, para garantir a segurança do paciente.

Na Tabela 2 elencamos as medicações de risco baixo (verde), risco possível (laranja) e alto risco (vermelho) de prolongamento do intervalo QT e ocorrência de TdP. Assim, sempre que possível, deve-se preferir as medicações adicionais de baixo risco, pois tanto a HCQ como a AZ já são listadas como de alto risco para ocorrência de TdP.

Alguns medicamentos podem aumentar o risco por outros mecanismos ou de maneira indireta, como no caso da hipocalemia induzida por diuréticos. A lista completa de interações medicamentosa deve ser checada diariamente pelo site crediblemeds.org. ${ }^{19}$

Em caso de ocorrência de arritmia ventricular ou TdP (Tabela 3) ${ }^{20,21 .}$

- Lidocaína é o antiarrítmico de escolha:

- Sulfato de magnésio

- Isoprotenerol em TdP mediado por bradicardia

- Marcapasso provisório para pacientes bradicárdicos, com $\mathrm{TdP}$ recorrente. A frequência cardíaca inicial deve ser programada para 90 bpm e os ajustes feitos de acordo com a resposta clinica do paciente.

- Suspensão imediata do uso de todas as medicações com potencial para prolongamento do intervalo QT.

\section{Conclusão}

O risco de arritmias fatais, facilitado pelo uso da HCQ e/

\begin{tabular}{|c|c|c|c|}
\hline & Alto risco & Moderado risco & $\begin{array}{c}\text { Baixo risco } \\
\text { ou NC }\end{array}$ \\
\hline Antiarrítmicos & $\begin{array}{l}\text { Amiodarona } \\
\text { Sotalol }\end{array}$ & Propafenona & $\begin{array}{l}\text { Lidocaína } \\
\text { Propranolol } \\
\text { Sulfato Mg } \\
\text { Isoproterenol }\end{array}$ \\
\hline \multirow[t]{3}{*}{ Antipsicóticos } & Haloperidol & Risperidona & Benzodiazepínico \\
\hline & Clorpromazina & Quetiapina & \\
\hline & Levomepromazine & $\begin{array}{l}\text { Prometazina } \\
\text { Olanzapina }\end{array}$ & \\
\hline \multirow[t]{2}{*}{ Sedativos } & Propofol & Dexmedetomidina & Midazolam \\
\hline & & & Fentanil \\
\hline $\begin{array}{l}\text { Antieméticos } \\
\text { e pró-cinéticos }\end{array}$ & $\begin{array}{l}\text { Ondansentrona } \\
\text { Domperidona } \\
\text { Bromoprida } \\
\text { Cisaprida }\end{array}$ & $\begin{array}{l}\text { Cimetidina } \\
\text { Granisetrona } \\
\text { Metoclopramida }\end{array}$ & Dimenidrato \\
\hline Antibióticos & Quinolonas & $\begin{array}{l}\text { Piperacilina- } \\
\text { tazobactam } \\
\text { Sulfametoxazole- } \\
\text { trimetropim }\end{array}$ & $\begin{array}{l}\text { Teicoplamida } \\
\text { Vancomicina }\end{array}$ \\
\hline Antifúngicos & Fluconazol & $\begin{array}{l}\text { Anfotericina } \\
\text { Itraconazol } \\
\text { Voriconazol }\end{array}$ & \\
\hline \multirow[t]{2}{*}{$\begin{array}{l}\text { Inibidores de } \\
\text { bomba de prótons }\end{array}$} & & Pantoprazol & \\
\hline & & $\begin{array}{l}\text { Omeprazol } \\
\text { Esomeprazol } \\
\text { Lanzoprazol }\end{array}$ & \\
\hline \multirow[t]{2}{*}{ Antialérgicos } & & Prometazina & Fexofenadina \\
\hline & & $\begin{array}{l}\text { Hidroxizina } \\
\text { Difenidramina }\end{array}$ & Loratadina \\
\hline Pandemia & $\begin{array}{l}\text { Cloroquina } \\
\text { Azitromicina }\end{array}$ & & Oseltamivir \\
\hline Broncodilatadores & & $\begin{array}{l}\text { Salbutamol } \\
\text { Fenoterol } \\
\text { Formoterol } \\
\text { Terbutalina }\end{array}$ & \\
\hline Anticolinesterázicos & Donepezila & Galantamina & \\
\hline Antidepressivos & $\begin{array}{l}\text { Citalopram } \\
\text { Escitalopram }\end{array}$ & $\begin{array}{c}\text { Fluoxetina } \\
\text { Paroxetina } \\
\text { Mirtazapina } \\
\text { Tricíclicos } \\
\text { Sertralina } \\
\text { Venlafaxina }\end{array}$ & \\
\hline Outros & $\begin{array}{l}\text { Cilostazol } \\
\text { Metadona } \\
\text { Tramadol }\end{array}$ & Loperamida & Fenitoína \\
\hline \multicolumn{4}{|l|}{ Cuidados especiais } \\
\hline Diuréticos & Cuidado & com espoliação de e & trólitos \\
\hline
\end{tabular}
ou AZ, em pacientes com infecção por COVID-19, ou em
Tabela 2 - Lista de medicações a serem evitadas (em vermelho e laranja)

NC-Não classificada, ou seja, sem evidência de prolongar o intervalo QT com base nos estudos publicados.

outras situações diárias fora da pandemia com medicações que tenham potencial para prolongamento do intervalo QT, pode ser minimizado com a aplicação de protocolos de conduta que auxiliem o profissional de saúde na decisão pela prescrição e manutenção do tratamento. 


\section{Ponto de Vista}

\begin{tabular}{l} 
Tabela 3 - Manejo Farmacológico da arritmia ventricular e/ou TdP \\
\hline Lidocaína \\
Dose de ataque - 1,0 a $1,5 \mathrm{mg} / \mathrm{kg}$ IV com doses repetidas em bolus de $0,5-$ \\
$0,75 \mathrm{mg} / \mathrm{kg}$ em bolus até $3 \mathrm{mg} / \mathrm{kg}$. \\
Manutenção - $20 \mathrm{mcg} / \mathrm{kg} / \mathrm{min}$ IV. \\
Sulfato de magnésio \\
2 a $4 \mathrm{~g}$ IV \\
Isoprotenerol \\
Dose de ataque: 1 a $2 \mathrm{mcg}$ IV. \\
Manutenção; $0,15 \mathrm{mcg} / \mathrm{min}$ e titular até $0,3 \mathrm{mcg} / \mathrm{min}$ de acordo com a resposta \\
ou necessidade clínica.
\end{tabular}

\section{Contribuição dos autores}

Concepção e desenho da pesquisa e Obtenção de dados: Wu TC; Redação do manuscrito: Wu TC, Sacilotto L, Darrieux FCC, Pisani CF, Hachul DT; Revisão crítica do manuscrito quanto ao conteúdo intelectual importante: Wu

\section{Referências}

1. Zhu N, Zhang D, Wang W, Li X, Yang B, Song J, et al. A Novel Coronavirus from Patients with Pneumonia in China, 2019. N Engl J Med. 2020 02;382(8):727-33.

2. worldometers. https://www.worldometers.info/coronavirus/[Cited in 2020, April 03] Available from: https://www.worldometers.info/ coronavirus/.

3. Wang M, Cao R, Zhang L, Yang X, Liu J, Xu M, et al. Remdesivir and chloroquine effectively inhibit the recently emerged novel coronavirus (2019-nCoV) in vitro. Cell Res. 2020 03;30(3):269-71.

4. Vincent MJ, Bergeron E, Benjannet S, Erickson BR, Rollin PE, Ksiazek TG, et al. Chloroquine is a potent inhibitor of SARS coronavirus infection and spread. Virol J. 2005 Aug; 2:69.

5. Wang D, Hu B, Hu C, Zhu F, Liu X, Zhang J, et al. Clinical Characteristics of 138 Hospitalized Patients With 2019 Novel Coronavirus-Infected Pneumonia in Wuhan, China. JAMA. 2020; 323(11):1061-9.

6. U.S. Food and Drug Administration. (FDA) Coronavirus Disease 2019 (COVID - 19) [Cited in 2020 March 28]. Available from: https://www.fda. gov/news-events/press-announcements/coronavirus-covid-19-updatedaily-roundup-march-30-2020.

7. Agência Nacional de Vigilância Sanitária. Anvisa. Covid-19: liberada pesquisa com hidroxicloroquina. [Citado em 27 março 2020] Disponível em:http://portal.anvisa.gov.br/

8. Gautret P, Lagier JC, Parola P, Hoang VT, Meddeb L, Mailhe M, et al. Hydroxychloroquine and azithromycin as a treatment of COVID-19: results of an open-label non-randomized clinical trial. Int J Antimicrob Agents. 2020 Mar:105949.

9. Bacharier LB, Guilbert TW, Mauger DT, Boehmer S, Beigelman A, Fitzpatrick AM, et al. Early Administration of Azithromycin and Prevention of Severe Lower Respiratory Tract Illnesses in Preschool Children With a History of Such Illnesses: A Randomized Clinical Trial. JAMA. 2015 Nov;314(19):2034-44.

10. Guo D, Cai Y, Chai D, Liang B, Bai N, Wang R. The cardiotoxicity of macrolides: a systematic review. Pharmazie. 2010 Sep;65(9):631-40.
TC, Sacilotto L, Darrieux FCC, Pisani CF, Melo SL, Hachul DT, Scanavacca M.

\section{Potencial conflito de interesses}

Os autores declaram não haver conflito de interesses pertinentes.

\section{Fontes de financiamento}

O presente estudo não contou com fontes de financiamento externas.

\section{Vinculação acadêmica}

Não há vinculação deste estudo a programas de pósgraduação.

\section{Aprovação ética e consentimento informado}

Este artigo não contém estudos com humanos ou animais realizados por nenhum dos autores.

11. Chen CY, Wang FL, Lin CC. Chronic hydroxychloroquine use associated with QT prolongation and refractory ventricular arrhythmia. Clin Toxicol (Phila). 2006;44(2):173-5.

12. Tisdale JE, Jaynes HA, Kingery JR, Mourad NA, Trujillo TN, Overholser BR, etal. Development and validation of a risk score to predict QT interval prolongation in hospitalized patients. Circ Cardi.ovasc Qual Outcomes. 2013 Jul;6(4):479-87.

13. Giudicessi JR, Noseworthy PA, Friedman PA, Ackerman MJ. Urgent Guidance for Navigating and Circumventing the QTC Prolonging and Torsadogenic potential of possible pharmacotherapies for (COVID-19). Mayo Clin Proc.xxx 2020: 1-9. [In Press]

14. Malviya A. Ventricular Arrhythmia Risk Due to HydroxychloroquineAzithromycin Treatment For COVID-19. Indian Heart]. 2020 Apr 27 [Epub ahead of print]

15. Priori SG, Schwartz PJ, Napolitano C, Bloise R, Ronchetti E, Grillo M, et al. Risk stratification in the long-QT syndrome. N Engl J Med. 2003 May;348(19):1866-74.

16. Johnson JN, Ackerman MJ. QTc: how long is too long? Br J Sports Med. 2009 Sep;43(9):657-62.

17. Postema PG, Wilde AA. The measurement of the QT interval. Curr Cardiol Rev. 2014 Aug; 10(3):287-94.

18. El-Sherif N, Turitto G, Boutjdir M. Acquired Long QT Syndrome and Electrophysiology of Torsade de Pointes. Arrhythm Electrophysiol Rev. 2019;8(2):122-30.

19. CredibleMeds. Quick search for drugs on the QTdrugs lists- [Cited in $2020 \mathrm{Apr}$ 20]. [Available from: crediblemeds.org.

20. Panchal AR, Berg KM, Kudenchuk PJ, Del Rios M, Hirsch KG, Link MS, et al. 2018 American HeartAssociation Focused Update on Advanced Cardiovascular Life Support Use of Antiarrhythmic Drugs During and Immediately After Cardiac Arrest: An Update to the American Heart Association Guidelines for Cardiopulmonary Resuscitation and Emergency Cardiovascular Care. Circulation. 201812;138(23):e740-e9.

21. Sorajja D, Munger TM, Shen Win-Kuang S. Optimal antiarrhythmic drug therapy for electrical storm. J Biomed Res. 2015;29(1):20-34. 